\title{
DIE TRISTANNOVELLE: EINE DRAMATISCHE BURLESKE
}

\section{Erwin Theodor Rosenthal}

\begin{abstract}
"Die Worte schienen ihm durchaus nicht zuzuströmen; für einen, dessen bürgerlicher Beruf das Schreiben ist, kam er jämmerlich langsam von der Stelle, und wer ihn sah, musste zu der Anschauung gelangen, dass ein Schriftsteller ein Mann ist, dem das Schreiben schwerer fällt als allen anderen Leuten" (251) (1). Auf diese Weise wird im Tristan der Poet Detlev Spinell gekennzeichnet, auf dessen Tisch "beständig, für jeden sichtbar, der sein Zimmer betrat, das Buch (lag), das er geschrieben hatte" (224). Schon hier ist die komisch-satirische Konzeption, die Thomas Mann in der Erzählung der Künstlerproblematik angedeihen lässt, einwandfrei zu erkennen, und obgleich der Antagonismus Kunst-Leben jahrelang die Hauptthematik des Dichters geblieben ist, tritt er gewöhnlich nicht unter dem Vorzeichen der spielerischen Ironie auf, die hier das ganze Werk beherrscht. Spinell ist ein JugendstilDichter und hält sich im Sanatorium 'Einfried', das der ganzen Novelle als Darstellungsort dient, nicht zur Ausheilung irgendwelcher Krankheiten auf, sondern lediglich "des Stiles wegen" Das Werk aus seiner Feder "war ein Roman von mässigem Umfange, mit einer vollkommen verwirrenden Umschlagzeichnung versehen und gedruckt auf einer Art von Kaffeesiebpapier mit Buchstaben, von denen ein jeder aussah wie eine gotische Kathedrale" (224). Die Gesellschaft lacht über diesen Spinell; er selbst lacht nie.
\end{abstract}

Die Lebensstimmung der Jugendstilzeit, dieser überreifen, überzüchteten und extrem-verfeinerten Stilrichtung der grossbürgerlichen Dekadenz ist vom Dichter oft beschrieben worden (man denke an das Bäder-Hotel und die dortige Gesellschaft im Tod in Venedig), doch erscheint der satirische Zug, so wie er im Tristan Form annimmt, erst

(1) - Der Text wird geboten nach Thomas Mann, Gesammelte Werke in zwölf Bänden, S. Fischer Verlag, Band VIII, Seite 216-262; die eingeklammerten Ziffern geben die Seitenzahlen an. Aus der Tristan-Forschung beanspruchi für diese Arbeit eine entscheidende Bedeutung: Wolfdietrich Rasch, Thomas Manns Erzählung 'Tristan'; in: Zuz deutschen Literatur seit der Jahrhundertwende, Stuttgart, 1967. 
später wieder (Die Bekenntnisse des Hochstaplers Felix Krull) Von dieser Sicht aus soll die Novelle verstanden werden, als eine schmunzelnde Kritik an dieser überzüchteten Verfeinerung, der der Dichter iibrigens selbst häufig erlag. Er weiss darum, und es stellt einen typischen Zug Mannscher Erzählweise dar, Selbstironie der Behandlung des Themas beizumischen .

Am 13. Februar 1901 kündigt der Dichter seinem Bruder Heinrich die Zusammenstellung des Novellenbandes an, der dann (allerdings in abgeänderter Form) 1903 erscheint und den Namen Tristan nach der Ttitelgeschichte tragen wird. Im Brief steht unsere Erzählung noch an fünfter Stelle und der infrage kommende Passus lautet: "Was den Novellenband angeht, so wird er ja ein schmales Ding, das mir nur eine vorläufige kleine Namensauffrischung und etwas Taschengeld eintragen soll. Eine Burleske, die ich in Arbeit habe, und die wahrscheinlich "Tristan" heissen wird. (Das ist echt! Eine Burleske, die "Tristan" heisst!)" (2). Und es geschah sicher nicht im Scherz, dass 'Thomas Mann dem Bruder gegenüber die Novelle als Burleske bezeichnete, denn wenngleich er das Possenhafte nicht als Leitfaden dieses kleinen Meisterwerks gesehen haben wollte, entspricht die drastisch-ironische Behandlung des Themas sehr wohl dieser Bezeichnung.

Es wird nun versucht werden, diese kleine Textstelle aus dem Brief an den Bruder Heinrich als Interpretationsschlüssel zu verwenden und dadurch einen Zugang zur Erzählung zu gewinnen. Sie ist in zwölf Teile gegliedert (die in der Gesamtausgabe numeriert sind): Das Sanatorium (1); Herr Klöterjahn und Gabriele Klöterjahn (2); Gabrieles Gesundheit, von Klöterjahn dargestellt (3); Der Schriftsteller Spinell (4); Gabriele nimmt Notiz von Spinell (5); Gabriele und Spinell in geselligem Zusammensein (6); Selbstdarstellung Gabrieles (7); Spinell und Gabriele allein; sie spielt Klavier (8); Klöterjahn sen. tritt wieder mit Gabrieles Söhnchen auf (9); Gabrieles Geschichte von Spinell in einem Brief dargestellt (10); Spinell unterliegt in der Konfrontation mit Klöterjahn; Gabriele stirbt (11); Spinell flieht vor Klöterjahn jr. (12).

Die dramatische Gruppierung dieser Teile ergibt einen Aufbau, der es ermöglicht, die Novelle als Einortdrama zu sehen, in dem stufenweise das ganze Geschehen linear aufgerollt wird. Die Situation wird in den ersten vier Teilen ausgebreitet (Exposition), und zwar mittels einer deutlichen Trennung zwischen dem ersten und den

(2) - Thomas Mann, Briefe 1889-1932, S. Fischer Verlag, 1962, S. 26. 
nachfolgenden Teilen, denn zuerst kommen der Raum (das Sanatorium) und dessen Funktion zur Geltung und erst dann werden die Hauptfiguren eingeführt. In den nächsten drei Teilen (5-7) wird der Knoten der Handlung geschürzt (Konflikt). Im achten Teil wird der Umschlag auf die Höhe getrieben (Peripetie), worauf sich in den weiteren drei 'Teilen die Lösung ergibt (Katastrophe) und die Flucht Spinells im zwölften Teil den Ausklang (Epilog) darstellt. Dieser sehr systematische Aufbau (bei Trennung der Raumdarstellung von der Exposi.ion erscheinen drei Einzelszenen und drei Gruppen von jeweils drei Szenen) ist also nach dem Muster des klassischen Dramas erfolgt und hier erscheint eine weitere, überaus feine Ironie: eine Prosaburleske, die sich auf ein Schema des klassischen Dramas reduzieren lässt! Das entspricht dem Ausruf: "Eine Burleske, die Tristan heisst!"

"Hier ist 'Einfried', das Sanatorium! Weiss und geradlinig liegt es mit seinem langgestreckten Hauptgebäude und seinem Seitenflügel inmitten des weissen Gartens, der mit Grotten, Laubengängen und kleinen Pavillons aus Baumrinde ergötzlich ausgestattet ist, und hinter seinen Schieferdächern ragen tannengrün, massig und weichzerklüftet die Berge himmelan" (216). Diese Sätze bezeichnen den Anfang der Novelle. Der Erzähler stellt in anmutig geschwungenem Bogen und einer sorgfältig abschattierten Sprache ein Stilideal der Zeit dar, das durch Grotten und Pavillons aus Baumrinde genugsam angedeutet wird. Die Berge, die in einer mächtigen Szenerie zu Himmel ragen, ïihren nicht, wie das in der Romantik der Fall war, dem Menschen seine Kleinheit vor Augen, sondern nehmen den Charakter einer theatralisch effektvollen Kulisse an, vor der er erst richtig ihrer Bedeutung inne wird. Die Natur, beherrscht und 'eingefriedet', wie der Name des Sanatoriums es schon andeutet, dient ihm zur Schaustellung, zu der er die dekorative Ornamentik benötigt. Das alles weist auf den Jugendstil, in den sich die Hauptfiguren Gabriele und Spinell mühelos einfügen lassen, und der sowobl in der "verwirrenden Umschlagzeichnung" des Romans als auch in dem 'Typus der fragilen, überschlanken Gabriele, der 'femme-enfant', zum Vorschein kommt. Dass Thomas Mann dieses Stilideal bespöttelt, ist sowohl aus dem Tonfall der ganzen Novelle zu erkennen, als auch an Einzelheiten, so daran, dass 'Einfried' eine unverhohlene Anspielung auf 'Wahnfried' ist, das Palais, das sich Richard Wagner in Bayreuth hat bauen lassen. Das Sanatorium als Bühne für die Handlung beweist andrerseits den unfehlbaren Griff Thomas Manns, immer dann wenn es darum geht, eine Szene aufzubauen: hier konnten sich Menschen aus verschiedenen Gegenden und heterogenster Provenienz treffen, sie mussten nur über genügend Geld verfügen und somit einer Schicht angehören, deren disparateste Vertreter zueinandergeführt werden konnten. Spinell, der 
sich mit einem "verwischten Schatten" seines Schönheitsideals begnügt, um ihm "nicht plump und wirklichkeitsgierig ins Gesicht" (230) zu starren, ist der ausgesuchteste Typus dieser Kunstvorstellungen und auch die Grotten, Laubengänge und Pavillone aus Baumrinde gemahnen an eine verklärte Wirklichkeit, die der Poet, durch seine effektheischenden Sätze nicht darstellen, sondern erahnen lassen will!

In diesem ersten Teil werden die leitenden Personen der Anstalt eingeführt, die sich unter anderm - und auch das ist burlesk! durch ihre Namen auszeichnen. Doktor Leander ist der Direktor. Er, der den Namen des feurigen Liebhabers der Hero trägt, ist durch "die Wissenschaft gekältet, gehärtet und mit stillem nachsichtigem Pessimismus erfüllt" (216), während der zweite Arzt folgendermassen vorgestellt wird: "Aber er heisst Müller und ist überhaupt nicht der Rede wert" (218). Haushälterin in 'Einfried' ist Fräulein von Osterloh (mann bemerke die hinter der Silbe 'loh' verborgenen Flammen) und "auf ihren Wangen. glüht in zwei runden, karmoisinroten Flecken die unauslöschliche Hoffnung, dereinst Frau Doktor Leander zu werden. " (216). Auch Patienten erscheinen, oft mit andeutenden Namen bedacht, so die dumme Magistratsrätin Spatz und die Pastorin Höhlenrauch, "die neunzehn Kinder zur Welt gebracht hat und keines Gedankens mehr fähig ist" (217). Seines lauten Wesens wegen passt Klöterjahn gut $\mathrm{zu}$ seinem Namen, doch wer die Frau so nennen wollte, "verdiente die Peitsche" (232).

Die nächsten drei Teile machen die Exposition aus. Es werden die Hauptpersonen in den Blickpunkt gebracht. Da erscheint zuerst Gabriele, die sich durch "schwache Grazie und zarten Liebreiz" (218) auszeichnet, eingeführt von ihrem Mann, dem Grosskaufmann Klöterjahn. Sie leidet 'an der Luftröhre' und soll hier die Kräfte wiedergewinnen, die sie seit der Geburt ihres kräftigen, gesunden und wohlgeratenen Sohnes verloren hatf. Diese Lungenkranke, denn das ist sie, bezieht ihre zauberhaft-liebreiche Anziehungskraft gerade aus der dekadent-morbiden Zerbrechlichkeit, die ihr eigen ist. Das stellen alle fest; sogar der Kutscher, der sie ins Sanatorium fährt. Er, "ein roher, unbewusster Mann ohne Feingefühl", wagt in ihrem Beisein kaum zu sprechen, "vor ohnmächtiger Behutsamkeit", ein Gefühl, das er sogar seinen Pferden mitteilt, die denn auch der porzellanfigürlichen Gabriele "voll Besorgnis" nachschauen, und so diesem "ängstlichen Vorgang" (218) eine besonders ironische Note verleihen.

Klöterjahn, Gabrieles Mann, repräsentiert recht eigentlich den Lebenskult, der die Zeit um die Jahrhundertwende entscheidend mit- 
bestimmt. Seine lärmende, strotzende, übergesunde Vitalität wird unterstrichen, und nicht bloss des Kontrastes zu seiner Gattin wegen, sondern well die vital Schwachen, so glaubte es die Überzeugung der Zeit, wenn sie sich Anstrengungen hingaben, die ihre Kräfte überforderten, es mit dem Tode zu büssen hatten, während die robuste Gesundheit, das überquellende Leben, alle Gefährdungen trotzend überstand. Gerade im Gegensatz zu dem rotbäckigen Klöterjahn, der ein Ideal der Zeit repräsentiert, wirkt die schwächlich-delikate Frau, die "sich instinktiv der starken Männlichkeit Klöterjahns zugewandt hatte" (3) rührend und gemahnt an das Gefährliche des Lebens. Sie wird, sich überanstrengend, dem Leben entrissen werden, doch Mann und Sohn, die sich auf der gesunden Hälfte des Lebens befinden und Spinell, der ästhetisch-delikat, jeder Gewalt abhold und sehr fürsorglich waltet, wenn es um die eigene Gesundheit geht, führen unangefochten ihr Dasein weiter. Das erscheint schon anfangs angedeutet. Es handelt sich tatsächlich um einen Verweis auf Ideale der Zeit, und wenn der nationalistische Historiker Treitschke die Entwicklung des preussischen Staates allegorisiert: "Dieser waffenstarke Staat, wie er so dastand, eine jugendlich unreife Gestalt, knochig und sehnig, Kraft und Trotz im Blicke, aber unschön, ohne die Fülle der. Formen, aller Anmut, alles Adels bar" gibt er eine Darstellung der Helden der Zeit, die in einigen Zügen auch auf Klöterjahn anwendbar ist (4). So erscheint das Bild des Vitalen als Symbol des reinen Bios, der physischen Gesundheit, der das Seelenleben zu fehlen scheint und für den geistige Werte nichts oder wenig bedeuten. Das Ganze aber, das Gesamtleben, entsteht aus der Verbindung des Starken mit dem Zarten, dem Geisteswerte viel bedeuten, dem das Leben bekannt und die Todesnähe bewusst ist. Sehr oft vertritt diese Empfindung eine weibliche Gestalt, die sich jetzt der früheren Darstellung der Frau als niederes Wesen ohne Seele entgegenstellt. Fontanes Effi Briest ist, zumindest nach ihrer Trennung von Innstetten, ein solcher Typus: in Rilkes frühen Gedichten finden sich dauernde Anspielungen auf solche Gestalten, die das Liebliche und den Traum verbinden, seine Darstellung in der Weise von Liebe und Tod fördert ein ähnliches Bild zutage:

"Nur im Sch'afe schaut man solchen Staat und solche Feste solcher Frauen: ihre kleinste Geste ist eine Falte, fallend in Brokat. Sie bauen Stunden auf aus silbernen Gesprächen, und manchmal heben sie die Hände so - , und du musst meinen,

(3) - Wolfdietrich Rasch, a.a.O., S. 149.

(4) - Apud Hamann-Hermand, Gründerzeit, Nymphenburger, S. 52. 
dass sie irgendwo, wo du nicht hinreichst, sanfte Rosen brächen, die du nicht siehst" (5).

Diese Frauen sind die Geheimnisvollen, Namenlosen, sie sind "liebende "lagende zagende wesen" (6), wie auch Gabriele dargestellt wird, "weich und ermüdet", mit "schönen, blassen Händen", die "der unsäglichen Zartheit, Süssigkeit und Mattigkeit des Köpfchens" entsprechen (219). So sieht sie Spinell, der Jugendstil-Poet, und dieses Bild erregt seine Phantasie über alle Massen. Er selbst wird nicht als sehr attraktiv beschrieben: "Herr Spinell besass eine gewölbte, poröse Oberlippe römischen Charakters, grosse, kariöse Zähne und Füsse von seltenem Umfange. Einer der Herren ( .) hatte ihn 'der verweste Säugling' getauft. "(223). Er stellt in allem den absoluten Gegensatz. zu Klöterjahn dar, ist ein "wunderlicher Kauz", der manchmal in "ästhetischen Zustand" verfällt, verbringt seine Tage mit Briefschreiben, obwohl er "höchst selten" welche empfängt (224). Der also Charakterisierte scheint nun wirklich nicht auserkoren, den Tristan für dic überzarte Isolde abzugeben. Doch so ist es, nur wird das Todesmotiv, um es schon vorwegzunehmen, lediglich symbolisch auf Isolde/Gabriele anwendbar sein. Tristan/Spinell erleidet keinen Liebestod.

In den folgenden drei Teilen wird der Knoten der Handlung geschürzt. Zuerst wird das gegenseitige Interesse bezeugt, indem sich Gabriele bei Dr. Leander nach dem Namen Spinells und dieser bei demselben sich nach der Benennung der Neuankömmlinge erkundigt. Leander, der nicht viel von Spinell hält, antwortet ihr: “ .nicht Spinelli, gnädige Frau. Nein, er ist kein Italiener, sondern bloss aus Lemberg gebürtig, soviel ich weiss. "(224). Auf die Benennung des nun wirklich auf die Persönlichkeit des Grosskaufmanns geprägten Namens bekommt er zu hören: "Wie heisst der Mann? fragte Herr Spinell. - Klöterjahn heissen sie! sagte Doktor Leander und ging seiner Wege" (226) Spinell hält den Namen für inadäquat für das überaus zierliche und gebrechliche Geschöpf, das zu seinem Träger geworden war Daher besteht er auf ihrem Mädchennamen, da, wer sie "Frau Klöterijahn nennen wollte, die Peitsche verdiente. (. .) Es ist Barbarei und Niedertracht, wenn man die Sitte so weit treibt, auf Sie den Namen Ihres Herrn Gemahls zu übertragen" (232). Auch hier ertönt wieder die komische UUbertreibung, die das ganze Wesen des

(5) - Rainer Maria Rilke, Die Weise von Liebe und Tod des Cornets Christoph Rilke, Insel, I, S. 23.

(6) - Stefan George, zit. bei Jost Hermand, Undinen-Zauber in Wissenschaft als Dialog, Metzler, S. 20. 
Mannes charakterisiert, der "des Stiles wegen" im Sanatorium lebt, dessen "Helligkeit und Härte" ihm "Haltung und Würde" verleihen soll, und, genau wie das Frühaufstehen, zu seiner "sittlichen Hebung" beiträgt (228). Dieser Spinell ist der lebensfeindliche Typ, der duckmäuserische Ästhet, der gar nicht das Bedürfnis fühlt, dem Leben zu huldigen; der einen 'verwischte Schatten' der Realität vorzieht, denn dieser regt seine Phantasie an, ohne ihn später zu enttäuschen. Und wenn er in ästhetische Zustände verfällt, so ist sein Lieblingsausdruck der Ausruf: "wie schön, o Gott, wie schön!" $(224,230,234)$.

Spinell, dieser "ganz wunderliche Kauz" (231) aktualisiert im Bericht seiner Haltung und Selbstbezwingung (das Leben im Sanatorium des 'Stiles wegen'; das frühmorgendliche Aufstehn als moralische Anstrengung) sowie seiner Eindrücke (der 'halbe Blick' auf die schöne Frau und das ästhetische Zurückschaudern vor dem Namen Klöterjahn) sein eigenes Bewusstsein, das eben nicht Ich und die Wirklichkeit reflektiert, sondern in dem das Ich sich eitel spiegelt. Es ist der Stolz auf seine überragende Intelligenz, der in seinen nicht eben häufigen Suaden deutlich zutage tritt, und auch hier nimmt der Erzähler die Gelegenheit wahr zur Feststellung der burleskhaften Ironisierung, bei der Beschreibung seiner Person zum Beispiel, die "in einem exaltierten Lächeln kariöse Zähne sehen lässt" oder durch die unfreiwillige Komik einer Replik, die beweist, dass seine Darstellung gründlich missverstanden wurde, als er Gabrieles Gesicht, durch seine Phantasie engelhaft gestaltet und ins Ubersinnliche gehoben, als etwas beschreibt, auf dem er "verweilen möchte, nicht Minuten, nicht Stunden, sondern mein ganzes Leben lang, mich ganz darin verlieren und alles Irdische Jarüber vergessen. ", worauf er von Herrn Klöterjahns Gattin gesagt bekommt: "Ja, ja Herr Spinell. Nur dass Fräulein von Osterloh doch ziemlich abstehende Ohren hat" (231).

Doch Gabriele, deren krankhaft-zarte Züge noch von dem "kleinen, seltsamen Äderchen, das sich blassblau und kränklich in der Klarheit und Makellosigkeit dieser wie durchsichtigen Stirn verzweigte" (219) hervogehoben wurden, machte sich Gedanken über ihre neue Bekanntschaft, hauptsächlich weil es Spinell gelungen war, ihre Neugier anzufachen dahingehend, dass es sie verlangte, ihr eigenes Wesen näher kennenzulernen. In der Unterhaltung mit ihm erinnert sie sich früherer Stunden, Vater Eckhof wird erwähnt mit seinem Geigenspiel, was wiederum Spinell zu seinem 'ästhetischen' Ausruf hinreisst, und ihn immer stärker in Gabrieles Augen positiv gegen ihren Mann absetzt, auf den der zur Sentenz gewordene Satz: "Cet homme, as:urément, 
n'aime pas la musique" (7) hätte mit Fug und Recht angewendet werden können. Hier erscheinen allerdings auch Bemerkungen, die Aspekte der spezifischen Gedankenwelt Thomas Manns enthüllen, so dass Rasch sicher im Recht ist, wenn er in Spinell "ein durch komische Zutaten wirksam maskiertes, als verzerrtes Spiegelbild gegebenes Selbstporträt des Autors" sieht (8) So behaupter Spinell, "dass ein Geschlecht mit praktischen, bürgerlichen und trockenen Traditionen sich gegen das Ende noch einmal durch die Kunst verklärt" (234), für jeden Leser ein klarer Hinweis auf die Buddenbrooks.

An dieser Stelle erzählt nun Gabriele ihre Geschichte, in der sie mit sechs Freundinnen häkelnd am Springbrunnen im Garten hinter ihrem alten Hause sitzt, eine Geschichte, die, von Spinell mit dem Ausruf "wie schön! Gott, hören Sie, wie schön!" bedacht, später von ihm umgeformt, grosse Bedeutung für die Auslegung seiner Persönlichkeit gewinnen soll. Hier nimmt die seelische Verführung, der Gabriele dann erliegen wird, ihren Anfang. Die Hervorhebung der Gesundheit und Ähnlichkeit von Vater und Sohn Klöterjahn unterstreicht den Kontrast zur Labilität Gabrieles, die den Gatten in Erinnerung behält, als einen Menschen, der ihr "hart und wohlmeinend auf die Schulter" zu schlagen scheint, während sie es vorzieht, "in Schwäche und Gehobenheit auf den Wolkenpfühlen zu ruhen, die Herr Spinell ihr dienend bereitete" (237).

Der Höhepunkt in den Beziehungen Gabriele/Spinell und deren Umschlag erfolgt im achten Teil. Es wird ein Tag beschrieben, an dem Vbermut in 'Einfried' herrscht, sogar "die Herren mit den unbeherrschten Beinen waren ganz ausser Rand und Band" (238). Den meisten Patienten wurde nämlich erlaubt, unter der sachkundigen Führung Doktor Leanders, eine Schlittenpartie zu unternehmen, nur die Schwerkranken mussten zurückbleiben und ausserdem nahmen auch der Poet, die Magistratsrätin, die Pastorin Höhlenrauch sowie Gabriele Klöteriahn nicht an dem Ausflug teil. Erst in den Abendstunden war die "Expedition" zurückzuerwarten und so trafen sich am Nachmittag Gabriele und Spinell im Konversationszimmer, anfänglich im Beisein der Rätin Spatz. Hier befreit Spincll sich von der Selbstbefangenheit, in die ihn vorher jede direkte Aussprache mit Gabriele gestürzt hatte. Er bestrickt sie mit der wiederholten Bitte, seinen ästhetischen Wünschen um Musik zu willfahren, er besteht auf ihrem Klavierspiel, das ihr von den Ärzten ausdrücklich verboten worden war, da es ihrer Gesundheit schadete. Er geht sie um das Spiel der 'Nocturnes' von

(7) - J. B. P. Molière, Amphitryon (1668)

(8) - Wolfdietrich Rasch, a.a.O., S. 169. 
Chopin an, selbst nachdem sie ihm deutlich gemacht hatte, dass es ihr schaden könnte: "Wenn Sie fürchten sich zu schaden, gnädige Frau, so lassen sie die Schönheit tot und stumm, die unter Ihren Fingern laut werden möchte" (242). Damit bricht er den letzten Widerstand. Schon Jieses 'Nocturne' spielt auf die Nacht an, die sie symbolisch von jetzt an umgeben wird, es "fehlt nur, dass ich die Kerzen anzünde. " (242). Die Katastrophe bahnt sich an, die in dem Masse komödienhaft konzipiert ist, als die Beteiligten sich nicht durch Mangel des Bewusstseins, sondern durch Mangel an Bewusstsein provozieren: Spinell dadurch, dass er der labilen Gesundheit Gabrieles nicht achtet, sie durch ihre mangelnde Widerstandskraft gegen seine Wünsche. Sie gab an, nur das eine Stück spielen zu wollen, doch es wurden drei, und dann erhob sie sich, "aber nur, um auf dem oberen Klavierdeckel nach neuen Noten zu suchen" (243). Später folgt Tristan und Isolde, die Partitur der Oper, die schon nach dem Vorspiel die Rätin Spatz, bei der "die Langeweile jenen Grad erreicht hatte, wo sie des Menschen Antlitz entstellt" (244) vertrieben hatte und deren Todesmystik nun die Lungenkranke und den zitternden Ästheten zueinander treibt: "Du Isolde, Tristan ich, nicht mehr Tristan, nicht mehr Isolde" (246).

Es ist der grotesk wirkende Literat, der Gabriele in die künstlich erzeugte Sphäre hebt, ihr das Zusammenspiel der Rhythmen erklärt und - sie lautlos anbetend - dann im schwarzen Gehrock vor ihr kniet. Es ist der Liebesgesang Wagners, der Beiden alles gleichzeitig bietet: "Schweigen und Ton, Tod und Gesang" (9). Das Ganze ist eme literarische Parodie der Wagnerschwelgereien der Jugendstilzeit. Thomas Mann selbst hat in seinem Vortrag Leiden und Grösse Richard Wagners den Tristantext des Komponisten in engem Zusammenhang mit Schopenhauer, mit Schlegels Lucinde und den Hymnen an die Nacht des Novalis gesehen und erwähnt, dass er bei der Lektüre des Schlegelschen Romans "in dem Liebesdialog zwischen Lucinde und Julius die ekstatische Replik anstrich. und an den Rand schrieb Tristan" (10). Die Nacht, die für diese Oper, die Wagner selbst als die "tiefe Kunst des tönenden Schweigens" bezeichnet hatte, atmosphärenbestimmend wirkt, erscheint in der Novelle gänzlich umgestaltet, so wie auch eine dahinweisende Bemerkung Schlegels parodistisch umgeformt wird. Der Liebesgesang stellt nicht ein Vorspiel zur Liebesertüllung dar und die im ironischen Falsett dargebotene Auslegung täuscht den Leser der Novelle nicht. So hiess es bei Schlegel: "O ew'ge Sehnsucht! - Doch endlich wird des Tages fruchtlos Sehnen, eitles

(9) - Hans Mayer, Tristans Schweigen in Zur deutschen Klassik und Romantik, Pfullingen, 1963, S. 351.

(10) - Thomas Mann, a.a.O., Band IX, S. 400. 
Blenden sinken und erlöschen, und eine grosse Liebesnacht sich ewig ruhig fühlen" (11). Und bei Thomas Mann erscheint in Parodie: "Trennen konnte sie des Tages tiickisches Blendwerk, doch seine prahlende Lüge vermochte die Nachtsichtigen nicht mehr zu täuschen, seit die Kraft des Zaubertrankes ihnen den Blick geweiht. Wer liebend des Todes Nacht und ihr süsses Geheimnis erschaute (12), dem blieb im Wahn des Lichtes ein einziges Sehnen, die Sehnsucht hin zur heiligen Nacht, der ewigen, wahren, der einsmachenden. " (245). Nachdem das Spiel abgeklungen war, umgab sie tiefe Stille. Er lag vor ihr auf dem Boden, "seine Schultern zuckten" und sie "sass, die Hände im Schosse, vornübergelehnt, vom Klavier abgewandt, und blickte auf ihn" (248). Nach dem Sehnsuchtsmotiv waren sie Erfüllung und Liebestod gewärtig, doch "aus weiter Ferne her näherten sich Schellenklappern, Peitschenknall und das Ineinanderklingen menschlicher Stimmen" (248).

In den folgenden drei Teilen ergibt sich die Lösung des Knotens; die Katastrophe bricht ein. Zwei Tage nach der Schlittenpartie musste Gabriele/Isolde sich legen und "gab. . Blut von sich" (248). Ihr Zustand wurde von Doktor Leander als unheilbar erkannt, der die Behandlung, wie stets in solchen Fällen, Doktor Müller übertrug, von dem Herr Klöterjahn samt dem kleinen Anton nach 'Einfried' bestellt wurden. Mit ihrer Ankunft vollzieht sich der Rückzug Spinells in sein Zimmer, wo der agressive Brief entsteht, den er Klöterjahn schreibt, eine Mikronovelle innerhalb der ganzen Erzählung, in der das erscheint, was Thomas Mann selbst die "melancholisch-skeptische Kritik am Künstlertum überhaupt, unter dem moralischen und menschlichen Gesichtspunkt" genannt hat (13). "Herr Spinell sass in seinem Zimmer und 'arbeitete" "(250). So heisst es anfangs und wenn das Tätigkeitswort in Anführungszeichen erscheint, so doch weil der Erzähler Zweifel an der Gültigkeit dieser Arbeit hat. In der Tat ist hier eine Prise Selbstironie dem Stoff beigemischt, die an die Tirade erinnert, die sich Thomas Mann 1907 in der autobiographischen Skizze Im Spiegel leistet: "Ein Dichter ist, kurz gesagt, ein auf allen Gebieten ernsthaf er Tätigkeit unbedingt unbrauchbarer, einzig auf Allotria bedachter, dem Staate nicht nur nicht nützlicher, sondern sogar aufsässig gesinnter Kumpan. ein innerlich kindischer, zur Aus-

(11) - Zitiert nach Thomas Mann, a.a.O., Band IX, S. 400.

(12) - Dieser Passus parodiert offensichtlich das Wagnerzitat aus Tristan und Isolde: "Wer des Todes Nacht / liebend erschaut, / wem sie ihr tief / Geheimnis vertraut." S. 20 .

(13) - In Blätter der Thomas Mann Gesellschaft, Zürich, Nr. 6, 1966, 
schwefung geneigter und in jedem Betrachte anrïchiger Scharlatan, der von der Gesel'schaft nichts anderes solte zu gewärtigen ha':en. als stille Verachtung" (14).

Ironisiert wird gleicherweise der starke Einfluss des Jugendstils auf den Poeten, der ihn auf Bogen schreiben lässt, in deren "linkem oberen Winkel unter einer verzwickt gezeichneten Landschaft der Name Detlev Spinell in völlig neuartigen Lettern zu lesen war" (250) und der auch verantwortlich ist für die äussere Haltung des Briefschreibers und den Eindruck, den Stil und Schreibweise erwecken: "Mit zwei Fingerspitzen hielt er eins der sonderbaren Flaumhärchen an seiner Wange erfasst und drehte Viertelstunden lang daran, indem er ins Leere starrte und nicht um eine Zeile vorwärtsrückte, schrieb dann ein paar zierliche Wörter und stockte aufs neue. Andererseits muss man zugeben, dass das, was schliesslich zustande kam, den Eindruck der Glätte und Lebhaftigkeit erweckte, wenn es auch inhaltlich einen wunderlichen, fragwürdigen und oft sogar unverständlichen Charakter trug" (251). Ganz im Geist des Jugendstils stellt die preziöse Stilisierung sich dar, mit der Spinell ein von Gabriele erwecktes Bild aus ihrer Jugenderinnerung umformt. Hier erscheinen, wie Wolfdietrich Rasch überzeugend beweist, genaue Anlehnungen an Zeichnungen und Beschreibungen aus der Zeit um 1900 und Spinell ist sich scheinbar gar nicht bewusst, dass er mit eigener Interpretation die dürftigen Angaben aus Gabriele Eckhofs Jugendzeit bereichert. Er sagt nämlich, dass er eine "kurze, unsäglich empörende" Geschichte erzählen möchte, "ohne Kommentar, ohne Anklage und Urteil" (251). Doch der Vergleich mit der Beschreibung der Gabriele mach` seine jugendstilmässige Auslegung überdeutlich. Gabriele hatte absolut naiv berichtet: "Ja, ich habe all die Jahre in lieber Erinnerung; besonders den Garten, unseren Garten, hinterm Hause. Er war jämmerlich verwildert und verwuchert und von zerbröckelten, bemoosten Mauern eingeschlossen. Dieses aus der Erinnerung geformte Abbild wird folgendermassen verwandelt: "Erinnern Sie sich des Gartens, mein Herr, des alten, verwucherten Gartens hinter dem grauen Patrizierhause? Das grüne Moos spross in den Fugen der verwitterten Mauern, die seine verträumte Wildnis umschlossen" (252). So wird das ganze Bild umgestaltet und aus den häkelnden Freundinnen Gabrieles werden singende Jungfrauen, die ihre Blicke auf den Wasserstrahl des Springbrunnens richten, "und ihre leisen, hellen Stimmen umschweben seinen schlanken Tanz" (252). Diese Szene begruindet in Spinells Brief die Empörung, die er gegen den "plebejischen Gourmand", den "Bauer mit Geschmack" (253) Klöterjahn verspürt. Aus seiner Sicht heraus ist es dessen Schuld, dass

(14) - Thomas Mann, a.a.O., Band XI, S. 332-333. 
dem erwähnten zarten Bilde eine "Fortsetzung der Gemeinheit und des hässlichen Leidens" (252) gegeben wurde, und - besonders gesehmacklos in seinen Augen - dass Gabriele diesen "ordinären Namen (254) trug, und die "müde, scheue und in erhabener Unbrauchbarkeit blühende Schönheit des Todes" (254) in den grauen Alltag erniedrigt wurde. Man bemerkt hier die Verklärung des Todes, der als in "blühender Schönheit" prangend gesehen wird und man erkennt sofort den wahren Grund der Spinellschen Begeisterung für Gabriele. Er liebt sie nicht, auch will er sie nicht besitzen, wie dieser 'ordinäre' Klöterjahn; er verspürt sich durch sie ästhetisch gekitzelt und es ficht ihn nicht an, dass sie die Erfüllung seiner Wünsche mit dem Leben bezahlen muss. Das lebensferne und feindliche Element seiner Anschauung und Begeisterung ist somit treffend gekennzeichnet.

Die Katastrophe, Gabrieles Tod, tritt ein, noch während Herr Klöterjahn in Spinells Zimmer, mit dessen Brief in der Hand, den Dichter mit einer Schimpfkanonade aus dem Bereich des 'gesunden Lebens' abkanzelt und der Poet, direkt konfrontiert mit seinem Gegner, gar nicht die Argumente mehr zur Hand hat, die seine brieflich geäusserte Empörung unterstï zen könnten. Den agressiven Vorwürfen gegen den banausischen Klöterịahn wird vollends das Gewicht genommen, wenn sich Spinell in der Konfrontation "lächelnd, entschuldigend und beinahe demütig" (256) benimmt und Gabrieles Gatte sogar, anhand einer Beschreibung der Handschrift des Poeten indirekt den von ihm vertretenen Zeitstil in seiner ganzen Schwäche verspottet: "Sie schreiben eine Hand, die miserabel ist. Auf den ersten Blick scheint es ganz sauber, aber bei Licht besehen ist es voller Lücken und Zittrigkeiten" (247) Ja, der 'wunderliche Kauz' wird heruntergerissen nach Strich und Faden und dessen geistreiche Formulierungen werden mit unschön gewählten Worten abgetan. $\mathrm{Da}$ ist vom "Herz in den Hosen haben" die Rede und von "in die Pfanne hauen", Spinell wird als 'Esel' und 'hinterlistiger Idiot' gekennzeichnet, und da er dasteht, "hilflos. wie ein grosser, kläglicher, grauhaariger Schuliunge" (257) bekommt er erzählt, wie das war mit dem Bild der 'sieben Jungfrauen' im Garten: “'Sie sangen' Punkt. Sie sangen gar nicht! Sie strickten . Ausserdem sprachen sie, soviel ich verstanden habe, von einem Rezept für Kartoffelpuffer" (259). Weiter konnte die Desillusionierung nicht getrieben werden!

Als auf diese Art das preziös aufgebaute Jugendzeitbild zusammenbricht, klopft es und Klöterjahn wird von der Magistratsrätin Spatz an das Sterbebett der überzarten Gattin gerufen. Genau hier nimmt der Epilog seinen Anfang, denn während Klöterjahn zu seiner sterbenden Frau eilt, schliesst Spinell die Tür, "betrachtet sich im Spiegel" 
und "nimmt einen Kognak zu sich, was kein Mensch ihm verdenken konnte" (260). Ihn ficht der Tod von Frau Klöterjahn nicht an, und ausserdem: "man ist nicht geschaffen für so plumpe Erlebnisse wie dieses da" (261). Einen plötzlichen Entschluss fassend, nimmt er seinen Hut, tritt aus dem Zimmer und geht in den Garten. "Er schritt in tiefem Sinnen' (261). Die Natur bietet wieder das stilmässige Aussehen der Zeit, die Nachmittagsbeleuchtung hebt die Grotten, Laubengänge und Pavillons noch hervor, und Spinell summt eine Musik vor sich hin: das Sehnsuchtsmotiv aus Tristan und Isolde. Doch als er gerade wieder im Begriff war in 'ästhetische Zustände' zu verfallen, erkennt er einen Kinderwagen im Garten, der gerade auf seinem Wege stand, und darin "sass Anton Klöterjahn der Jüngere, sass Gabriele Eckhofs dicker Sohn!" (262) Er liess Detlev nicht vorüber, denn er begann "zu lachen and zu jubeln', "er kreischte vor unerklärlicher Lust" (262) und sein "animalisches Wohlbefinden" schlägt den Poeten in die Flucht, da er "gefolgt von dem Jubilieren des kleinen Klöterjahn" spürt, "dass er innerlich davonläuft" (262).

Diese dem Gelächter preisgegebene Gestalt, die unter gewissen äusseren und inneren Aspekten einer Beschreibung des Schriftstellers Arthur Holitscher (Das unruhige Asien, Worauf wartest du?, Lebensgeschichte eines Rebellen) sehr ähnelt (15) ist die Hauptgestal: dieser Burleske, und wahrlich die wirklich grotesk-komische Figur. Dasselbe kann nicht von Gabriele gesagt werden, wie auch nicht von Klöterjahn, dessen biedere Züge und banausische Verhaltensweisen unfreiwillige Komik vermitteln, die aber nicht komisch an sich wirken. Gleichviel entsteht im Leser der Eindruck des Burleskhaften, weil die Züge der Jugendstilepoche so stark nachgezogen und gegen das Natürliche abgesetzt werden. Das Parodistische des jugendstilmässig - dekorativen Schönheitskultes, das ihn als absolut leer, einseitig und dekadent decouvriert und Thomas Manns ironische Distanzierung von diesem Zeitstil beinhaltet, ermöglicht die burlesk-satirische Behandlung und erklärt die Ausführungen und die Benennung in der anfangs erwähnten Briefstelle . 